\title{
Studies the Reliability and Availability Characteristics of Two Different System under Preventive Maintenance
}

\author{
Khaled Moh. El-said \\ Dep.of Math., \\ Faculty of science, \\ Helwan University, \\ P.O.Box 11795, Cairo
}

\author{
Nareman Raghb \\ Dept. of Math., \\ Faculty of Science, \\ Helwan University, Cairo
}

\begin{abstract}
The research studies the availability and reliability to two systems which different due to the effect of preventive maintenance (PM), a two system analyses a two state with two type failure. The rate of failure is exponential distribution but the rate of repair is general distribution. The system resolved by supplementary technique and Laplace transforms depend on Complex imagine roots. Several measures of availability and (MTTF) of system are obtained. we analysis graphically to watch the impress of several system parameters in mean time failure and availability.
\end{abstract}

\section{Keywords}

Availability, Reliability, (MTTF), Laplace transforms (L.T), supplementary technique, preventive maintenance (PM).

\section{INTRODUCTION}

Reliability study of the repair problem of device is importance in our lifetime where it is used widely in the manufacturing system. So the systems repairable study is an important component in reliability analysis.. Many authors study availability, reliability and (MTTF) under P.M. Like [1] deals with cost-benefit of a two- unit cold standby system with twophase repair of the failed unit and preventive maintenance. The rate to failure and time to go PM are exponential while rate of repair and the time till go PM are general. [2] The author presents a two system having single unit in parallel which different because to the additional of the preventive maintenance. [3] Studied two parallel systems which every unit has two failure type where unit fails due to operating characteristics, so, the system go under preventive maintenance randomly. Rate of Failure are constant while the rate of PM \& repair are general.[4] This paper deals with an aero plane model; namely, a two-unit (non-identical) parallel system with dual mode of failures which preventive maintenance happen at random epochs. [5] Deals with a 2state repairable complex system of two failure types which solved by Laplace transform which rate of failure and repair of [type1, type2] are assumed as exponential distributed. [6] Study the availability and profit analysis of a repairable redundant 3-out-of-4 system with preventive [7] this paper show complex system where fail in n-mutually exclusive ways of total failure. [8] Study some reliability parameters of three states with failure environmental [9] the author tale about complex system consisting two subsystems where use supplementary technique \& Laplace Transform (L.T). [10] Talk about the system reliability where transform the basic equations of the model into integro-differential eq. and solve it using Supplementary variables. This research show comparison between two systems where they different

because of the additional of PM. the two systems resolved by partial differential eq. \& Laplace Transform where depend on Complex imagine roots then we show numerical results to analyze the impress of the various system parameters on reliability and system availability.

\section{SYSTEM DESCRIPTION}

The system is analyzed under following practical assumptions:

- The system unit contain of two state repairable units with two failure types.

- The system has two repair facilities, first for repairing type $1 \&$ the second for type 2 .

- After repairing a unit, it will work like a new state.

We suppose that preventive maintenance is provided to this system at random epochs when the system at state So. Through the P.M the system remains operating.

3. NOTATIONS AND SYSTEM STATES: $\lambda_{1} \& \lambda_{2} \quad$ Rate of failure of type $1 \&$ type 2

$\mu_{1} \& \mu_{2} \quad$ Rate of repair of type $1 \&$ type 2 $\mu_{3} \quad$ Constant rate for taking a unit into preventive maintenance

$\mu_{1}(x), \mu_{2}(y): \quad$ general repair of $S_{1}, S_{2}$ elapsed time of repair $\mathrm{x}$ and $\mathrm{y}$.

$\mu_{4}(\mathrm{x}): \quad$ General repair end of PM

$p_{j}(\tau) \quad$ Probability that the system is in state $S_{j}$ at time $t, j=0,1,2,3$

$0 \rightarrow$ normal state

$1 \rightarrow$ failed state of type 1 .

$2 \rightarrow$ failed state of type 2 .

$3 \rightarrow$ normal state and preventive maintenance.

$\mathrm{P}_{1}(\mathrm{x}, \tau) \& \mathrm{P}_{2}(\mathrm{y}, \tau) \quad$ Probability that the system in state $\mathrm{S}_{1} \& \mathrm{~S}_{2}$ at time $\tau$, and

under repair, elapsed time of repair is $\mathrm{x} \&$ y

$P_{3}(x, \tau): \quad \quad \quad \quad \quad \quad \quad \quad \quad$ robability that the system in state $S_{3}$ at time $\tau$, and under Preventive Maintenance, elapsed time of repair is $\mathrm{x}$

$\mathrm{p}_{\mathrm{j}}^{*}(\mathrm{~s}) \quad$ Laplace transform (L.T) of $\mathrm{p}_{\mathrm{j}}(\tau)$

A $(\tau): \quad$ functions of availability. 
$\mathrm{R}(\tau)$

MTTF: mean time failure.

Where Laplace transforms (L.T) of $\mathrm{p}_{j}(\tau)$ is:

$p_{j}^{*}(s)=\int_{0}^{\infty} e^{-S \tau} p_{j}(\tau)$

There are a relation between repair rate $\mu_{1}(\mathrm{x}), \mu_{2}(\mathrm{y})$ and their cumulative functions $\mathrm{F}(\mathrm{x}), \mathrm{F}(\mathrm{y})$, i.e.

First system with P.M

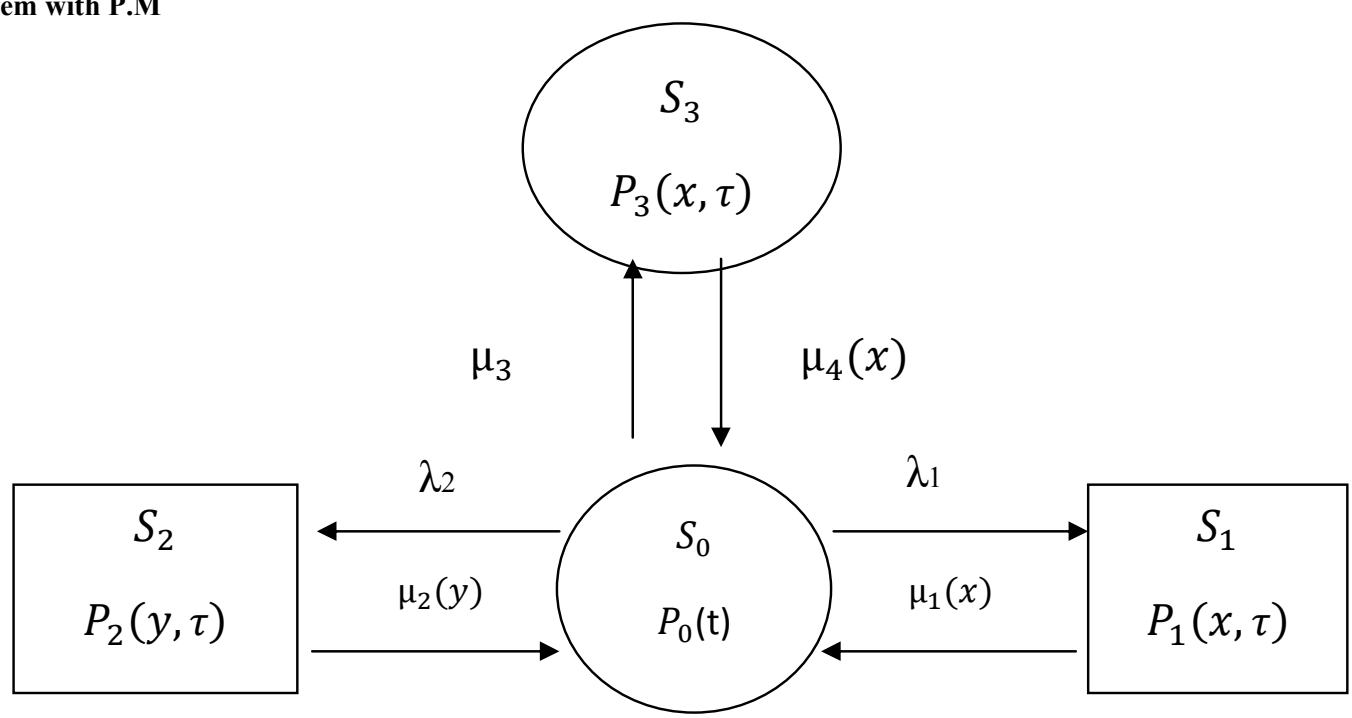

Figure1. System configuration diagram

\section{MATHEMATICAL MODEL DESCRIPTION}

This part showing the differential eq. for the system of figure (1) Transition states

$\left(\frac{\partial}{\partial t}+\lambda_{1}+\lambda_{2}+\mu_{3}\right) P_{0}(\tau)=$

$\int_{0}^{\infty} \mu_{1}(x) P_{1}(x, \tau) d x+\int_{0}^{\infty} \mu_{2}(y) P_{2}(y, \tau) d y+$

$\int_{0}^{\infty} \mu_{4}(x) P_{3}(x, \tau) d x$

$\left(\frac{\partial}{\partial t}+\frac{\partial}{\partial x}+\mu_{1}(x)\right) P_{1}(\mathrm{x}, \tau)=0$

$\left(\frac{\partial}{\partial t}+\frac{\partial}{\partial y}+\mu_{2}(y)\right) P_{2}(\mathrm{y}, \tau)=0$

$\left(\frac{\partial}{\partial t}+\frac{\partial}{\partial x}+\mu_{4}(x)\right) \mathbf{P}_{3}(\mathrm{x}, \tau)=0$

Initial conditions:

$\mathbf{P}_{\mathbf{j}}(0)=\left\{\begin{array}{lc}\mathbf{1} & \text { where } \mathbf{j}=\mathbf{0} \\ \mathbf{0} & \text { else }\end{array}\right.$

Boundary conditions

$$
\begin{aligned}
& P_{1}(0, \tau)=\lambda_{1} P_{0}(\tau) \\
& P_{2}(0, \tau)=\lambda_{2} P_{0}(\tau) \\
& P_{3}(0, \tau)=\mu_{3} P_{0}(\tau)
\end{aligned}
$$

\section{MODEL SOLUTION}

Use Laplace Transform (L.T) For Eq. (4-1) to (4-4) and Boundary conditions

$\left(\mathrm{s}+\lambda_{1}+\lambda_{2}+\mu_{3}\right) \mathrm{P}_{0}^{*}(\mathrm{~s})=$

$1+$

$\int_{0}^{\infty} \mu_{1}(\mathrm{x}) \mathrm{P}_{1}^{*}(\mathrm{x}, \mathrm{s}) \mathrm{dx} \int_{0}^{\infty} \mu_{2}(\mathrm{y}) \mathrm{P}_{2}^{*}(\mathrm{y}, \mathrm{s}) \mathrm{dy}+$

$\int_{0}^{\infty} \mu_{4}(\mathrm{x}) \mathrm{P}_{3}^{*}(\mathrm{x}, \mathrm{s}) \mathrm{dx}$

$$
\begin{aligned}
& \left(\mathrm{s}+\frac{\partial}{\partial \mathrm{x}}+\mu_{1}(\mathrm{x})\right) \mathrm{P}_{1}^{*}(\mathrm{x}, \mathrm{s})=0 \\
& \left(\mathrm{~s}+\frac{\partial}{\partial y}+\mu_{2}(\mathrm{y})\right) \mathrm{P}_{2}^{*}(\mathrm{y}, \mathrm{s})=0 \\
& \left(\mathrm{~s}+\frac{\partial}{\partial \mathrm{x}}+\mu_{4}(\mathrm{x})\right) \mathrm{P}_{3}^{*}(\mathrm{x}, \mathrm{s})=0
\end{aligned}
$$

With Boundary conditions

$$
\begin{aligned}
& \mathrm{P}_{1}^{*}(0, \mathrm{~s})=\lambda_{1} \mathrm{P}_{0}^{*}(\mathrm{~s}) \\
& \mathrm{P}_{2}^{*}(0, \mathrm{~s})=\lambda_{2} \mathrm{P}_{0}^{*}(\mathrm{~s}) \\
& \mathrm{P}_{2}^{*}(0, \mathrm{~s})=\mu_{3} \mathrm{P}_{0}^{*}(\mathrm{~s})
\end{aligned}
$$

$\mathrm{P}_{1}^{*}(\mathrm{x}, \mathrm{s})=\mathrm{P}_{1}^{*}(0, \mathrm{~s}) \mathrm{e}^{-\mathrm{sx}-\int_{0}^{\mathrm{x}} \mu_{1}(\mathrm{x}) \mathrm{dx}}$

$\mathrm{P}_{2}^{*}(\mathrm{y}, \mathrm{s})=\mathrm{P}_{2}^{*}(0, \mathrm{~s}) \mathrm{e}^{-\mathrm{s} y-\int_{0}^{\mathrm{y}} \mu_{2}(\mathrm{y}) \mathrm{dy}}$

$\mathrm{P}_{3}^{*}(\mathrm{x}, \mathrm{s})=\mathrm{P}_{3}^{*}(0, \mathrm{~s}) \mathrm{e}^{-\mathrm{s} x-\int_{0}^{\mathrm{x}} \mu_{4}(\mathrm{x}) \mathrm{dx}}$

Again Integrating by parts equations (5-8)-(5-10) using (5-5)-(5-7)

$\mathrm{P}_{1}^{*}(\mathrm{~s})=\int_{0}^{\infty} \mathrm{P}_{1}^{*}(\mathrm{x}, \mathrm{s}) \mathrm{dx}=\mathrm{P}_{1}^{*}(0, \mathrm{~s}) \mathrm{s}^{-1}\left\{1-\mathrm{F}_{1}^{*}(\mathrm{~s})\right\}$

$\therefore \mathrm{P}_{1}^{*}(\mathrm{~s})=\mathrm{P}_{0}^{*}(\mathrm{~s}) \mathrm{A}_{1}(\mathrm{~s})$

$\therefore \mathrm{P}_{2}^{*}(\mathrm{~s})=\mathrm{P}_{0}^{*}(\mathrm{~s}) \mathrm{A}_{2}(\mathrm{~s})$

$\therefore \mathrm{P}_{3}^{*}(\mathrm{~s})=\mathrm{P}_{0}^{*}(\mathrm{~s}) \mathrm{A}_{3}(\mathrm{~s})$

Where

$\mathrm{A}_{1}(\mathrm{~s})=\lambda_{1} \mathrm{~N}_{1}(\mathrm{~s})$

$A_{2}(s)=\lambda_{2} N_{2}(s)$

$A_{3}(s)=\mu_{3} N_{3}(s)$ 


$$
\begin{aligned}
& \mathrm{N}_{1}(\mathrm{~s})=\mathrm{s}^{-1}\left\{1-\mathrm{F}_{1}^{*}(\mathrm{~s})\right\} \\
& \mathrm{N}_{2}(\mathrm{~s})=\mathrm{s}^{-1}\left\{1-\mathrm{F}_{2}^{*}(\mathrm{~s})\right\} \\
& \mathrm{N}_{3}(\mathrm{~s})=\mathrm{s}^{-1}\left\{1-\mathrm{F}_{3}^{*}(\mathrm{~s})\right\}
\end{aligned}
$$

And

$F_{1}^{*}(s)=\int_{0}^{\infty} \mu_{1}(x) e^{-s x-\int_{0}^{x} \mu_{1}(x) d x} d x$

$\mathrm{F}_{2}^{*}(\mathrm{~s})=\int_{0}^{\infty} \mu_{2}(\mathrm{y}) \mathrm{e}^{-\mathrm{s} y-\int_{0}^{\mathrm{y}} \mu_{2}(\mathrm{y}) \mathrm{dy}} \mathrm{dy}$

$\mathrm{F}_{3}^{*}(\mathrm{~s})=\int_{0}^{\infty} \mu_{4}(\mathrm{x}) \mathrm{e}^{-\mathrm{s} x-\int_{0}^{\mathrm{x}} \mu_{4}(\mathrm{x}) \mathrm{dx}} \mathrm{dx}$

Also we have from equations (5-8)-(5-10)

using equations (5-5)-(5-7)

$\int_{0}^{\infty} \mathrm{P}_{1}^{*}(\mathrm{x}, \mathrm{s}) \mu_{1}(\mathrm{x}) \mathrm{dx}=\lambda_{1} \mathrm{P}_{0}^{*}(\mathrm{~s}) \mathrm{F}_{1}^{*}(\mathrm{~s})$

$\int_{0}^{\infty} \mathrm{P}_{2}^{*}(\mathrm{y}, \mathrm{s}) \mu_{2}(\mathrm{y}) \mathrm{dy}=\lambda_{2} \mathrm{P}_{0}^{*}(\mathrm{~s}) \mathrm{F}_{2}^{*}(\mathrm{~s})$

$\int_{0}^{\infty} \mathrm{P}_{3}^{*}(\mathrm{x}, \mathrm{s}) \mu_{4}(\mathrm{x}) \mathrm{dx}=\mu_{3} \mathrm{P}_{0}^{*}(\mathrm{~s}) \mathrm{F}_{3}^{*}(\mathrm{~s})$

Now from equations (5-14) \& (5-15) and (5-16) in (5-1) we get

$\mathrm{P}_{0}^{*}(\mathrm{~s})=\frac{1}{\mathrm{~s}+\lambda_{1}+\lambda_{2}+\mu_{3}-\lambda_{1} \mathrm{~F}_{1}^{*}(\mathrm{~s})-\lambda_{2} \mathrm{~F}_{2}^{*}(\mathrm{~s})-\mu_{3} \mathrm{~F}_{3}^{*}(\mathrm{~s})}=\frac{1}{\mathrm{~A}(\mathrm{~s})}$

Where

$\mathrm{A}(\mathrm{s})=\mathrm{s}+\lambda_{1}+\lambda_{2}+\mu_{3}-\lambda_{1} \mathrm{~F}_{1}^{*}(\mathrm{~s})-\lambda_{2} \mathrm{~F}_{2}^{*}(\mathrm{~s})-$ $\mu_{3} \mathrm{~F}_{3}^{*}(\mathrm{~s})$

\section{EVALUATION DOWN AND UP STATE AVAILABILITY BY LAPLACE TRANSFORMS}

The system probability in operable (up) and failed (down) state at time " $\tau$ " can be obtained by Laplace transform as:

$$
\begin{aligned}
& \mathrm{P}_{\mathrm{up}}^{*}(\mathrm{~s})=\mathrm{P}_{0}^{*}(\mathrm{~s})+\mathrm{P}_{3}^{*}(\mathrm{~s}) \\
& \mathrm{P}_{\text {down }}^{*}(\mathrm{~s})=1-\mathrm{P}_{\mathrm{up}}^{*}(\mathrm{~s})
\end{aligned}
$$

\section{PARTICULAR CASE}

In this section the up and down state availability, MTTF, the steady -state availability of the system have been evaluated, when repair times will be exponential distribution.

Setting

$$
\begin{aligned}
& \mathrm{F}_{1}^{*}(\mathrm{~s})=\frac{\mu_{1}}{\mathrm{~s}+\mu_{1}}, \quad \mathrm{~F}_{2}^{*}(\mathrm{~s})=\frac{\mu_{2}}{\mathrm{~s}+\mu_{2}}, \\
& \mathrm{~F}_{3}^{*}(\mathrm{~s})=\frac{\mu_{4}}{\mathrm{~s}+\mu_{4}}
\end{aligned}
$$$$
\text { In equations (5-11)-(5-13),(5-17) we gets }
$$

$\mathrm{P}_{0}^{*}(\mathrm{~s})$

$$
\left(s+\mu_{1}\right)\left(s+\mu_{2}\right)\left(s+\mu_{4}\right)
$$

$\left(s+\lambda_{1}+\lambda_{2}+\mu_{3}\right)\left(s+\mu_{1}\right)\left(s+\mu_{2}\right)\left(s+\mu_{4}\right)-\lambda_{1} \mu_{1}\left(s+\mu_{2}\right)\left(s+\mu_{4}\right)-\lambda_{2} \mu_{2}\left(s+\mu_{1}\right)\left(s+\mu_{4}\right)-\mu_{4} \mu_{3}\left(s+\mu_{1}\right)\left(s+\mu_{2}\right)$

$$
\mathrm{P}_{1}^{*}(\mathrm{~s})=
$$$$
\frac{\lambda_{1}\left(s+\mu_{2}\right)\left(s+\mu_{4}\right)}{\left(s+\lambda_{1}+\lambda_{2}+\mu_{3}\right)\left(s+\mu_{1}\right)\left(s+\mu_{2}\right)\left(s+\mu_{4}\right)-\lambda_{1} \mu_{1}\left(s+\mu_{2}\right)\left(s+\mu_{4}\right)-\lambda_{2} \mu_{2}\left(s+\mu_{1}\right)\left(s+\mu_{4}\right)-\mu_{4} \mu_{3}\left(s+\mu_{1}\right)\left(s+\mu_{2}\right)}
$$

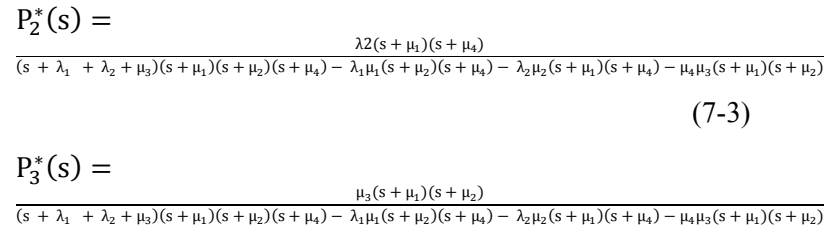

From equation (7-1),(7-4), one may get

$\mathrm{P}_{\mathrm{up}}^{*}(\mathrm{~s})=\mathrm{P}_{0}^{*}(\mathrm{~s})+\mathrm{P}_{3}^{*}(\mathrm{~s})$

$\mathrm{P}_{\text {up }}^{*}(\mathrm{~s})$

$=$

$\left(s+\mu_{1}\right)\left(s+\mu_{2}\right)\left(s+\mu_{4}\right)+\mu_{3}\left(s+\mu_{1}\right)\left(s+\mu_{2}\right)$

$\left(\mathrm{s}+\lambda 1+\lambda 2+\mu_{3}\right)\left(\mathrm{s}+\mu_{1}\right)\left(\mathrm{s}+\mu_{2}\right)\left(\mathrm{s}+\mu_{4}\right)-\lambda 1 \mu_{1}\left(\mathrm{~s}+\mu_{2}\right)\left(\mathrm{s}+\mu_{4}\right)-\lambda 2 \mu_{2}\left(\mathrm{~s}+\mu_{1}\right)\left(\mathrm{s}+\mu_{4}\right)-\mu_{4} \mu_{3}\left(\mathrm{~s}+\mu_{1}\right)\left(\mathrm{s}+\mu_{2}\right)$

$\mathrm{P}_{\mathrm{up}}^{*}(\mathrm{~s})=\frac{\mathrm{s}^{3}+\mathrm{As}^{2}+\mathrm{BS}+\mathrm{m}}{\mathrm{s}\left[\mathrm{s}^{3}+\mathrm{a}_{1} \mathrm{~s}^{2}+\mathrm{a}_{2} \mathrm{~s}+\mathrm{a}_{3}\right]}$

Where

$\mathrm{a}_{1}=\mu_{1}+\mu_{2}+\lambda_{1}+\lambda_{2}+\mu_{3}+\mu_{4}$

$\mathrm{a}_{2}=\mu_{1} \mu_{2}+\mu_{1} \lambda_{2}+\mu_{2} \lambda_{1}+\mu_{1} \mu_{3}+\mu_{1} \mu_{4}+\mu_{2} \mu_{3}+\mu_{2} \mu_{4}+$ $\mu_{4} \lambda_{2}+\mu_{4} \lambda_{1}$

$\mathrm{a}_{3}=\mu_{1} \mu_{2} \mu_{3}+\mu_{1} \mu_{2} \mu_{4}+\mu_{1} \mu_{4} \lambda_{2}+\mu_{2} \mu_{4} \lambda_{1}$

$\mathrm{A}=\mu_{1}+\mu_{4}+\mu_{2}+\mu_{3}$

$\mathrm{B}=\mu_{4} \mu_{2}+\mu_{1} \mu_{4}+\mu_{1} \mu_{2}+\mu_{3} \mu_{2}+\mu_{1} \mu_{3}$

$\mathrm{m}=\mu_{4} \mu_{1} \mu_{2}+\mu_{3} \mu_{1} \mu_{2}$

\subsection{Cubic equations roots have are 2 cases}

\subsubsection{First case}

$(\mathrm{D}>0) \quad[1$ root is real and 2 complex $]$

$\mathrm{P}_{\mathrm{up}}^{*}(\mathrm{~s})=\frac{\mathrm{s}^{3}+\mathrm{As}^{2}+\mathrm{BS}+\mathrm{m}}{\mathrm{s}\left(\mathrm{s}+\mathrm{A}_{1}-\mathrm{W}\right)\left(\mathrm{S}+\mathrm{A}_{1}+\mathrm{w}_{1}-\mathrm{i} \sqrt{3} \mathrm{v}_{1}\right)\left(\mathrm{S}+\mathrm{A}_{1}+\mathrm{w}_{1}+\mathrm{i} \sqrt{3} \mathrm{v}_{1}\right)}$

Where

$$
\begin{array}{lrl}
\mathrm{q}=\frac{3 \mathrm{a}_{2}-\mathrm{a}_{1}{ }^{2}}{9} & , \quad \mathrm{r}=\frac{9 \mathrm{a}_{1} \mathrm{a}_{2}-2 \mathrm{a}_{1}{ }^{3}-27 \mathrm{a}_{3}}{54} \\
\mathrm{D}=\mathrm{q}^{3}+\mathrm{r}^{2} & , & \mathrm{u}=(\mathrm{r}+\sqrt{\mathrm{D}})^{\frac{1}{3}} \\
\tau=(\mathrm{r}-\sqrt{\mathrm{D}})^{\frac{1}{3}} & \\
\mathrm{w}_{1}=\frac{(\mathrm{u}+\tau)}{2} & , & \mathrm{w}=(\mathrm{u}+\tau) \\
\mathrm{v}_{1}=\frac{(\mathrm{u}-\tau)}{2} & , & \mathrm{~A}_{1}=\frac{\mathrm{a}_{1}}{3}
\end{array}
$$

Applying inverse Laplace Transform for the eq., we obtain

$$
\begin{aligned}
& P(\tau)=\frac{m}{\left(A_{1}-w\right)\left(A_{1}^{2}+A_{1} w+w_{1}{ }^{2}+3 v_{1}{ }^{2}\right)} \\
& +\frac{\left(-A_{1}+w\right)^{3}+A\left(-A_{1}+w\right)^{2}+B\left(-A_{1}+w\right)+m}{\left(-A_{1}+w\right)\left(9 w_{1}{ }^{2}+3 v_{1}{ }^{2}\right)} e^{\left(-A_{1}+W\right) \tau} \\
& + \\
& \left\{\frac{2[\mathrm{PX}+3 \mathrm{HT}]\left[\cos \sqrt{3}\left(\mathrm{v}_{1}\right) \mathrm{t}\right]-2 \sqrt{3}[(\mathrm{H} \mathrm{X})-(\mathrm{TP})]\left(\sin \sqrt{3}\left(\mathrm{v}_{1}\right) \mathrm{t}\right)}{\mathrm{X}^{2}+3 \mathrm{~T}^{2}}\right\} \mathrm{e}^{\left(-\mathrm{A}_{1}-\mathrm{w}_{1}\right) \tau} \\
& \mathrm{P}=\left(-\mathrm{A}_{1}{ }^{3}-3 \mathrm{~A}_{1}{ }^{2} \mathrm{w}_{1}+9 \mathrm{~A}_{1} \mathrm{v}_{1}{ }^{2}-\mathrm{w}_{1}{ }^{3}+9 \mathrm{w}_{1} \mathrm{v}_{1}{ }^{2}-\mathrm{A}_{1} \mathrm{w}_{1}{ }^{2}\right)+ \\
& \mathrm{A}\left(\mathrm{A}_{1}{ }^{2}+\mathrm{A}_{1} \mathrm{~W}-3 \mathrm{v}_{1}{ }^{2}+\mathrm{w}_{1}{ }^{2}+\mathrm{B}\left(-\mathrm{A}_{1}-\mathrm{w}_{1}\right)+\mathrm{m}\right. \\
& \mathrm{H}=\left(6 \mathrm{~A}_{1} \mathrm{v}_{1} \mathrm{w}_{1}-3 \mathrm{v}_{1}{ }^{3}+3 \mathrm{w}_{1}{ }^{2} \mathrm{v}_{1}+3 \mathrm{~A}_{1}{ }^{2} \mathrm{v}_{1}-\mathrm{AA}_{1} \mathrm{v}-\mathrm{A} \mathrm{v}_{1} \mathrm{~W}\right. \\
& +\mathrm{Bv}_{1} \text { ) }
\end{aligned}
$$


$X=3 v^{2} w+6 v_{1}^{2} A_{1}$

$\mathrm{T}=3 \mathrm{wv}_{1} \mathrm{~A}_{1}+3 \mathrm{w}_{1}{ }^{2} \mathrm{v}-6 \mathrm{v}_{1}{ }^{3}$

- Reliability System and availability

System availability:

Availability of the system can be get from the relation

$$
\begin{aligned}
& A(\tau)=\frac{m}{\left(A_{1}-w\right)\left(A_{1}{ }^{2}+A_{1} w+w_{1}{ }^{2}+3 v_{1}{ }^{2}\right)} \\
& \quad+\frac{\left(-A_{1}+w\right)^{3}+A\left(-A_{1}+w\right)^{2}+B\left(-A_{1}+w\right)+m}{\left(-A_{1}+w\right)\left(9 w_{1}{ }^{2}+3 v_{1}{ }^{2}\right.} e^{\left(-A_{1}+W\right) \tau} \\
& +\quad\left\{\frac{2[P X+3 H T]\left[\cos \sqrt{3}\left(v_{1}\right) t\right]-2 \sqrt{3}[(H X)-(T P)]\left(\sin \sqrt{3}\left(v_{1}\right) t\right)}{X^{2}+3 T^{2}}\right\} e^{\left(-A_{1}-w_{1}\right) \tau}
\end{aligned}
$$

So we can obtain the steady - state availability (A) from the following relation

$A=\lim _{t \rightarrow \infty} A(\tau)$

$A=\frac{m}{\left(A_{1}-w\right)\left(A_{1}{ }^{2}+A_{1} w+w_{1}{ }^{2}+3 v_{1}{ }^{2}\right)}$

- $\quad$ The mean time failure (MTTF):

Taking all repairs zero in (7-4), mean time to failure of the system is obtained as

$\mathrm{MTTF}=\lim _{\mathrm{s} \rightarrow 0} \mathrm{P}_{\mathrm{up}}^{*}(\mathrm{~s})$

7.1.2. Second case $\quad \mathrm{D}<0 \quad$ [All roots are real and unequal]

$\mathrm{P}_{\mathrm{up}}^{*}(\mathrm{~s})=\frac{\mathrm{s}^{3}+A \mathrm{~s}^{2}+\mathrm{BS}+\mathrm{m}}{\mathrm{s}\left(\mathrm{s}+\mathrm{A}_{1}-\mathrm{w}_{0}\right)\left(\mathrm{S}+\mathrm{A}_{1}-\mathrm{w}_{2}\right)\left(\mathrm{S}+\mathrm{A}_{1}-\mathrm{v}_{2}\right)}$

Where

$$
\begin{array}{ll}
\mathrm{s}_{1}=\mathrm{w}_{0}-\frac{\mathrm{a}_{1}}{3} & , \quad \mathrm{~s}_{2}=\mathrm{w}_{2}-\frac{\mathrm{a}_{1}}{3} \\
\mathrm{~s}_{3}=\mathrm{v}_{2}-\frac{\mathrm{a}_{1}}{3} & , \quad \theta=\cos ^{-1} \frac{\mathrm{r}}{\sqrt{-\mathrm{q}^{3}}} \\
\mathrm{w}_{0}=2 \sqrt{-\mathrm{q}} \cos \left(\frac{\theta}{3}\right) & , \quad \mathrm{w}_{2}=2 \sqrt{-\mathrm{q}} \cos \left(\frac{\theta}{3}+120\right)
\end{array}
$$

$$
\mathrm{v}_{2}=2 \sqrt{-\mathrm{q}} \cos \left(\frac{\theta}{3}+240\right) \quad, \quad \mathrm{A}_{1}=\frac{\mathrm{a}_{1}}{3}
$$

By apply inverse Laplace transform

$$
\begin{aligned}
P_{u p}(\tau) & =\frac{m}{\left(A_{1}-w_{0}\right)\left(A_{1}^{2}-A_{1} w_{2}-A_{1} v_{2}+w_{2} v_{2}\right)} \\
& +\frac{\left(-A_{1}+w_{0}\right)^{3}+A\left(-A_{1}+w_{0}\right)^{2}+B\left(-A_{1}+w_{0}\right)+m}{\left(-A_{1}+w_{0}\right)\left(w_{0}^{2}-w_{0} w_{2}-w_{0} v_{2}+w_{2} v_{2}\right)} e^{\left(-A_{1}+w_{0}\right) \tau} \\
& +\frac{\left(-A_{1}+w_{2}\right)^{3}+A\left(-A_{1}+w_{2}\right)^{2}+B\left(-A_{1}+w_{2}\right)+m}{\left(-A_{1}+w_{2}\right)\left(w_{2}^{2}-w_{0} w_{2}-w_{2} v_{2}+w_{0} v_{2}\right)} e^{\left(-A_{1}+w_{2}\right) \tau} \\
& +\frac{\left(-A_{1}+v_{2}\right)^{3}+A\left(-A_{1}+v_{2}\right)^{2}+B\left(-A_{1}+v_{2}\right)+m}{\left(-A_{1}+v_{2}\right)\left(v_{2}^{2}-w_{0} v_{2}-w_{2} v_{2}+w_{0} w_{2}\right)} e^{\left(-A_{1}+v_{2}\right) \tau}
\end{aligned}
$$

- Reliability System and availability

System availability:

$$
\begin{aligned}
A(\tau)= & \frac{m}{\left(A_{1}-w_{0}\right)\left(A_{1}{ }^{2}-A_{1} w_{2}-A_{1} v_{2}+w_{2} v_{2}\right)} \\
& +\frac{\left(-A_{1}+w_{0}\right)^{3}+A\left(-A_{1}+w_{0}\right)^{2}+B\left(-A_{1}+w_{0}\right)+m}{\left(-A_{1}+w_{0}\right)\left(w_{0}{ }^{2}-w_{0} w_{2}-w_{0} v_{2}+w_{2} v_{2}\right)} e^{\left(-A_{1}+w_{0}\right) \tau} \\
& +\frac{\left(-A_{1}+w_{2}\right)^{3}+A\left(-A_{1}+w_{2}\right)^{2}+B\left(-A_{1}+w_{2}\right)+m}{\left(-A_{1}+w_{2}\right)\left(w_{2}^{2}-w_{0} w_{2}-w_{2} v_{2}+w_{0} v_{2}\right)} e^{\left(-A_{1}+w_{2}\right) \tau} \\
& +\frac{\left(-A_{1}+v_{2}\right)^{3}+A\left(-A_{1}+v_{2}\right)^{2}+B\left(-A_{1}+v_{2}\right)+m}{\left(-A_{1}+v_{2}\right)\left(v_{2}{ }^{2}-w_{0} v_{2}-w_{2} v_{2}+w_{0} w_{2}\right)} e^{\left(-A_{1}+v_{2}\right) \tau}
\end{aligned}
$$

And the steady - state availability are:

$A=\lim _{t \rightarrow \infty} A(\tau)$

$\mathrm{A}=\frac{\mathrm{m}}{\left(\mathrm{A}_{1}-\mathrm{w}_{0}\right)\left(\mathrm{A}_{1}{ }^{2}-\mathrm{A}_{1} \mathrm{w}_{2}-\mathrm{A}_{1} \mathrm{v}_{2}+\mathrm{w}_{2} \mathrm{v}_{2}\right)}$

- $\quad$ The mean time failure (MTTF):

Taking all repairs zero in (7-4), mean time to failure of the system is obtained as

MTTF $=\lim _{\mathrm{s} \rightarrow 0} \mathrm{P}_{\mathrm{up}}^{*}(\mathrm{~s})$

MTTF

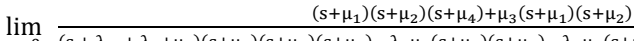

$\left(s+\lambda_{1}+\lambda_{2}+\mu_{3}\right)\left(s+\mu_{1}\right)\left(s+\mu_{2}\right)\left(s+\mu_{4}\right)-\lambda_{1} \mu_{1}\left(s+\mu_{2}\right)\left(s+\mu_{4}\right)-\lambda_{2} \mu_{2}\left(s+\mu_{1}\right)\left(s+\mu_{4}\right)-\mu_{4} \mu_{3}\left(s+\mu_{1}\right)\left(s+\mu_{2}\right)$

MTTF $=\frac{\mu_{4}+\mu_{3}}{\left(\lambda_{1}+\lambda_{2}\right)\left(\mu_{4}\right)}$

\section{System without PM}

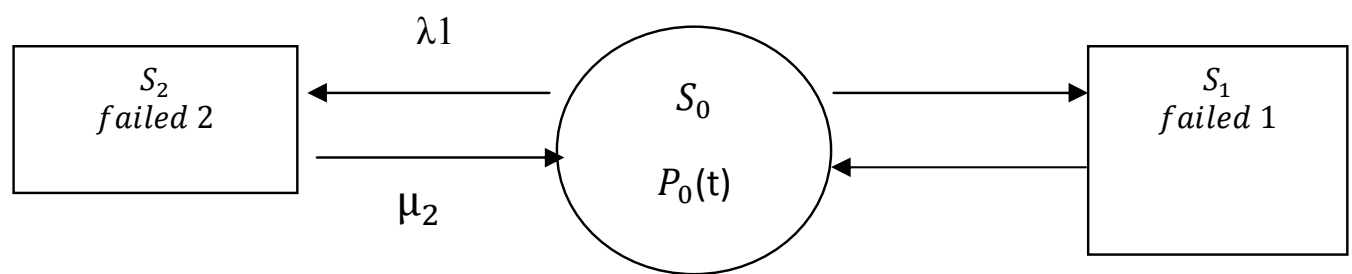

Figure2. System configuration diagram

\section{MATHEMATICAL MODEL DESCRIPTION}

This part showing the differential eq. for the system of Table (1) Transition states

$$
\begin{aligned}
& \left(\frac{\partial}{\partial t}+\lambda_{1}+\lambda_{2}\right) P_{0}(t)=\int_{0}^{\infty} \mu_{1}(x) P_{1}(x, t) d x+ \\
& \int_{0}^{\infty} \mu_{2}(x) P_{2}(y, t) d y \\
& \left(\frac{\partial}{\partial t}+\frac{\partial}{\partial x}+\mu_{1}(x)\right) P_{1}(x, t)=0
\end{aligned}
$$

$$
\left(\frac{\partial}{\partial \mathrm{t}}+\frac{\partial}{\partial \mathrm{y}}+\mu_{2}(\mathrm{y})\right) \mathrm{P}_{2}(\mathrm{y}, \mathrm{t})=0
$$

Initial conditions:

$P_{j}(0)=\left\{\begin{array}{lc}1 & \text { where } j=0 \\ 0 & \text { else }\end{array}\right.$

Boundary conditions

$\mathrm{P}_{1}(0, \mathrm{t})=\lambda_{1} \mathrm{P}_{0}(\mathrm{t}) \quad, \quad \mathrm{P}_{2}(0, \mathrm{t})=\lambda_{2} \mathrm{P}_{0}(\mathrm{t})$ 


\section{SOLUTION OF THE MODEL}

By taking Laplace transform for (8-1) to (8-3) and Boundary conditions

$\left(\mathrm{s}+\lambda_{1}+\lambda_{2}\right) \mathrm{P}_{0}^{*}(\mathrm{~s})=1+\int_{0}^{\infty} \mu_{1}(\mathrm{x}) \mathrm{P}_{1}^{*}(\mathrm{x}, \mathrm{s}) \mathrm{dx}+$

$\int_{0}^{\infty} \mu_{2}(y) P_{2}^{*}(y, s) d y$

$\left(\mathrm{s}+\frac{\partial}{\partial \mathrm{x}}+\mu_{1}(\mathrm{x})\right) \mathrm{P}_{1}^{*}(\mathrm{x}, \mathrm{s})=0$

$\left(\mathrm{s}+\frac{\partial}{\partial \mathrm{y}}+\mu_{2}(\mathrm{y})\right) \mathrm{P}_{2}^{*}(\mathrm{y}, \mathrm{s})=0$

With Boundary conditions

$\mathrm{P}_{1}^{*}(0, \mathrm{~s})=\lambda_{1} \mathrm{P}_{0}^{*}(\mathrm{~s})$

$\mathrm{P}_{2}^{*}(0, \mathrm{~s})=\lambda_{2} \mathrm{P}_{0}^{*}(\mathrm{~s})$

Integrating equations (9-2) \& (9-3)

$P_{1}^{*}(x, s)=P_{1}^{*}(0, s) e^{-s x-\int_{0}^{x} \mu_{1}(x) d x}$

$P_{2}^{*}(y, s)=P_{2}^{*}(0, s) e^{-s y-\int_{0}^{y} \mu_{2}(y) d y}$

Again Integrating by parts equations (9-6) \& (9-7) using (9-4) $\&(9-5)$

$\mathrm{P}_{1}^{*}(\mathrm{~s})=\int_{0}^{\infty} \mathrm{P}_{1}^{*}(\mathrm{x}, \mathrm{s}) \mathrm{dx}=\mathrm{P}_{1}^{*}(0, \mathrm{~s}) \mathrm{s}^{-1}\left\{1-\mathrm{F}_{1}^{*}(\mathrm{~s})\right\}$

$\therefore \mathrm{P}_{1}^{*}(\mathrm{~s})=\mathrm{P}_{0}^{*}(\mathrm{~s}) \mathrm{A}_{1}(\mathrm{~s})$

$\therefore \mathrm{P}_{2}^{*}(\mathrm{~s})=\mathrm{P}_{0}^{*}(\mathrm{~s}) \mathrm{A}_{2}(\mathrm{~s})$

Where

$$
\begin{aligned}
& \mathrm{A}_{1}(\mathrm{~s})=\lambda_{1} \mathrm{~N}_{1}(\mathrm{~s}) \\
& \mathrm{A}_{2}(\mathrm{~s})=\lambda_{2} \mathrm{~N}_{2}(\mathrm{~s}) \\
& \mathrm{N}_{1}(\mathrm{~s})=\mathrm{s}^{-1}\left\{1-\mathrm{F}_{1}^{*}(\mathrm{~s})\right\} \\
& \mathrm{N}_{2}(\mathrm{~s})=\mathrm{s}^{-1}\left\{1-\mathrm{F}_{2}^{*}(\mathrm{~s})\right\} \\
& \text { And } \\
& \mathrm{F}_{1}^{*}(\mathrm{~s})=\int_{0}^{\infty} \mu_{1}(\mathrm{x}) \mathrm{e}^{-\mathrm{sx}-\int_{0}^{\mathrm{x}} \mu_{1}(\mathrm{x}) \mathrm{dx}} \mathrm{dx}, \\
& \mathrm{F}_{2}^{*}(\mathrm{~s})=\int_{0}^{\infty} \mu_{2}(\mathrm{y}) \mathrm{e}^{-\mathrm{sy}-\int_{0}^{\mathrm{y}} \mu_{2}(\mathrm{y}) d y} \mathrm{dy}
\end{aligned}
$$

Also we have from equations (9-6) \& (9-7) using equations (9-4) \& (9-5)

$\int_{0}^{\infty} \mathrm{P}_{1}^{*}(\mathrm{x}, \mathrm{s}) \mu_{1}(\mathrm{x}) \mathrm{dx}=\lambda_{1} \mathrm{P}_{0}^{*}(\mathrm{~s}) \mathrm{F}_{1}^{*}(\mathrm{~s})$

$\int_{0}^{\infty} \mathrm{P}_{2}^{*}(\mathrm{y}, \mathrm{s}) \mu_{2}(\mathrm{y}) \mathrm{dy}=\lambda_{2} \mathrm{P}_{0}^{*}(\mathrm{~s}) \mathrm{F}_{2}^{*}(\mathrm{~s})$

Now from equations $(9-10) \&(9-11)$ in $(8-1)$ we get

$\mathrm{P}_{0}^{*}(\mathrm{~s})=\frac{1}{\mathrm{~s}+\lambda_{1}+\lambda_{2}-\lambda_{1} \mathrm{~F}_{1}^{*}(\mathrm{~s})-\lambda_{2} \mathrm{~F}_{2}^{*}(\mathrm{~s})}=\frac{1}{\mathrm{~A}(\mathrm{~s})}$

Where

$\mathrm{A}(\mathrm{s})=\mathrm{S}+\lambda_{1}+\lambda_{2}-\lambda_{1} \mathrm{~F}_{1}^{*}(\mathrm{~s})-\lambda_{2} \mathrm{~F}_{2}^{*}(\mathrm{~s})$

\section{EVALUATION DOWN AND UP STATE AVAILABILITY BY LAPLACE TRANSFORMS}

The system probability in operable (up) and failed (down) state at time " $\tau$ "can be obtained by Laplace transform as:

$$
\begin{aligned}
& \mathrm{P}_{\mathrm{up}}^{*}(\mathrm{~s})=\mathrm{P}_{0}^{*}(\mathrm{~s}) \\
& \mathrm{P}_{\text {down }}^{*}(\mathrm{~s})=1-\mathrm{P}_{\mathrm{up}}^{*}(\mathrm{~s})
\end{aligned}
$$

\section{PARTICULAR CASE}

In this section the up and down state availability, MTTF, the steady -state availability of the system have been evaluated, when repair times will be exponential distribution.

$$
\begin{aligned}
& \mathrm{F}_{1}^{*}(\mathrm{~s})=\frac{\mu_{1}}{\mathrm{~s}+\mu_{1}} \quad, \quad \mathrm{~F}_{2}^{*}(\mathrm{~s})=\frac{\mu_{2}}{s+\mu_{2}} \\
& \mathrm{P}_{0}^{*}(\mathrm{~s})=\frac{\left(\mathrm{s}+\mu_{1}\right)\left(s+\mu_{2}\right)}{\mathrm{s}\left(\mathrm{s}^{2}+\mathrm{s} \lambda_{1}+s \lambda_{2}+s \mu_{1}+s \mu_{2}+\lambda_{1} \mu_{2}+\lambda_{2} \mu_{1}+\mu_{1} \mu_{2}\right)} \\
& \mathrm{P}_{1}^{*}(\mathrm{~s})=\left\{\frac{\lambda_{1}\left(s+\mu_{2}\right)}{s\left(s^{2}+s \lambda_{1}+s \lambda_{2}+s \mu_{1}+s \mu_{2}+\lambda_{1} \mu_{2}+\lambda_{2} \mu_{1}+\mu_{1} \mu_{2}\right)}\right\} \\
& \mathrm{P}_{2}^{*}(\mathrm{~s})=\frac{\lambda_{2}\left(s+\mu_{1}\right)}{s\left(s^{2}+s \lambda_{1}+s \lambda_{2}+s \mu_{1}+s \mu_{2}+\lambda_{1} \mu_{2}+\lambda_{2} \mu_{1}+\mu_{1} \mu_{2}\right)}
\end{aligned}
$$

We know that

$\mathrm{P}_{\mathrm{up}}^{*}(\mathrm{~s})=\mathrm{P}_{0}^{*}(\mathrm{~s})$

$\mathrm{P}_{\mathrm{up}}^{*}(\mathrm{~s})=\frac{\left(\mathrm{s}+\mu_{1}\right)\left(\mathrm{s}+\mu_{2}\right)}{\mathrm{s}\left(\mathrm{s}^{2}+\mathrm{s} \lambda_{1}+\mathrm{s} \lambda_{2}+s \mu_{1}+s \mu_{2}+\lambda_{1} \mu_{2}+\lambda_{2} \mu_{1}+\mu_{1} \mu_{2}\right)}$

$\mathrm{P}_{\mathrm{up}}^{*}(\mathrm{~s})=\frac{\mathrm{s}^{2}+\mathrm{aS}+\mathrm{b}}{\mathrm{s}\left[\mathrm{s}^{2}+\mathrm{cS}+\mathrm{d}\right]}$

Where

$$
\begin{array}{lll}
\mathrm{b}=\mu_{1} \mu_{2} & , & \mathrm{a}=\mu_{1}+\mu_{2} \\
\mathrm{c}=\mu_{1}+\mu_{2}+\lambda 1+\lambda 2 & , & \mathrm{~d}=\mu_{1} \mu_{2}+\mu_{1} \lambda 2+\mu_{2} \lambda 1
\end{array}
$$

By using inverse of Laplace Transform (I.L.T) of eq., we obtain

$p_{\text {up }}(t)=\frac{1}{d}\left\{b+e^{-\frac{1}{2} t c}\left[(d-b) \cosh \left(\frac{1}{2} t \sqrt{c^{2}-4 d}\right)+\right.\right.$

$\left.\left.\frac{(2 \mathrm{ad}-\mathrm{dc}-\mathrm{cb}) \sinh \left(\frac{1}{2} \mathrm{t} \sqrt{\mathrm{c}^{2}-4 \mathrm{~d}}\right)}{\sqrt{\mathrm{c}^{2}-4 \mathrm{~d}}}\right]\right\}$

- Reliability System and availability

System availability:

Availability of the system can be get from the relation

$$
\begin{aligned}
& \mathrm{A}_{\text {up }}(\mathrm{t})=\frac{1}{\mathrm{~d}}\left\{\mathrm{~b}+\mathrm{e}^{-\frac{1}{2} \mathrm{tc}}\left[(\mathrm{d}-\mathrm{b}) \cosh \left(\frac{1}{2} \mathrm{t} \sqrt{\mathrm{c}^{2}-4 \mathrm{~d}}\right)+\right.\right. \\
& \left.\left.\frac{(2 \mathrm{ad}-\mathrm{dc}-\mathrm{cb}) \sinh \left(\frac{1}{2} \mathrm{t} \sqrt{\mathrm{c}^{2}-4 \mathrm{~d}}\right)}{\sqrt{\mathrm{c}^{2}-4 \mathrm{~d}}}\right]\right\}
\end{aligned}
$$

The steady - state availability can be obtained from the following relation

$$
A=\lim _{t \rightarrow \infty} A_{u p}(t)=\frac{\mu_{1} \mu_{2}}{\lambda_{1} \mu_{2}+\mu_{1} \mu_{2}+\lambda_{2} \mu_{1}}
$$

- Mean time to system failure

Taking all repairs zero in (11-4), mean time to failure of the system is obtained as

$$
\begin{aligned}
& \text { MTTF }=\lim _{s \rightarrow 0} P_{u p}^{*}(s) \\
& \text { MTTF }=\lim _{s \rightarrow 0} \frac{\left(s+\mu_{1}\right)\left(s+\mu_{2}\right)}{s\left(s^{2}+s \lambda_{1}+s \lambda_{2}+s \mu_{1}+s \mu_{2}+\lambda_{1} \mu_{2}+\lambda_{2} \mu_{1}+\mu_{1} \mu_{2}\right)} \\
& \text { MTTF }=\frac{1}{\left(\lambda_{1}+\lambda_{2}\right)}
\end{aligned}
$$

\section{NUMERICAL EXAMPLE}

To see the system behavior, we plot the steady -state availability for the models, against $\lambda 1$ keeping the other parameters fixed at

$\lambda_{2}=0.25, \quad \mu_{2}=0.3 \quad, \quad \mu_{1}=0.1 \quad, \mu_{3}=\mu_{4}=0.7$ 


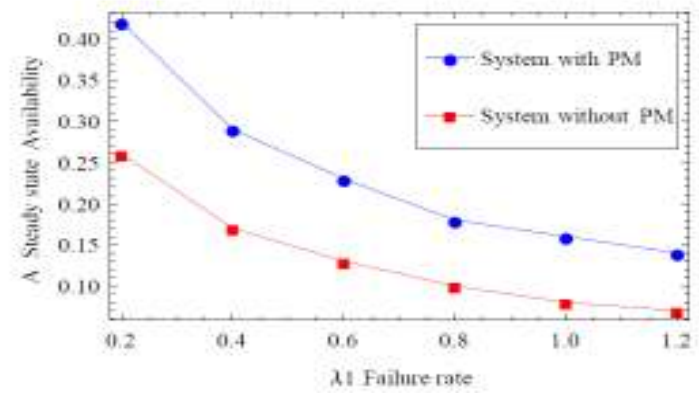

Fig 1.The Steady state Availability w.r.t. Failure Rate $\lambda 1$

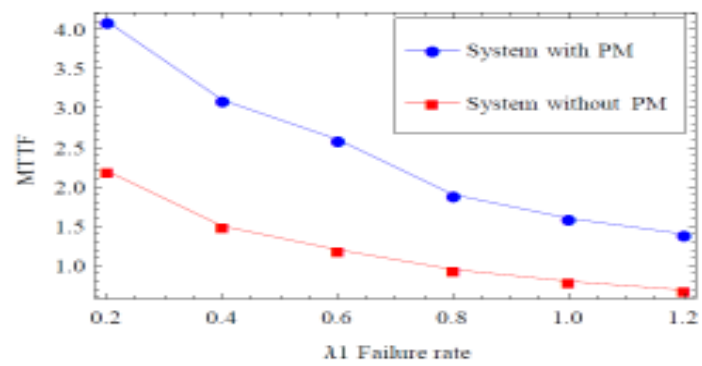

Fig 2.The mean time failure w.r.t. Failure Rate $\lambda 1$

\section{CONCLUSIONS}

- We use computer software, to plot system availability and MTTF in figure 1 and 2 respectively. It is noted that A decrease as $\lambda_{1}$ increases and MTTF decrease as $\lambda_{1}$ increases also the system with (P.M) is better than the system without (P.M).

\section{REFERENCES}

[1] Goel , L. R., Sharma, G. C., and Gupta, P., "Reliability analysis of a system with preventive maintenance inspection and two types of repair",Microelectronic Reliable, Vol. 26, pp. 492-433, 1986
[2] Goal, L.R .and Shrivastava, P., "Comparison of reliability characteristics of two (double component) systems with bivariate Exponential lifetimes". J.Systems Sci. Vol .23, PP.135-144, 1992

[3] Goel, L.R and Gupta, P., Stochastic analysis of a twounit parallel system with partial and catastrophic failures and preventive maintenance ",Microelectronic Reliable, Vol .24, PP.881-883, 1984

[4] Goel ,L.R and Gupta, P., "Analysis of two-engine aero plane model with two types of failure and preventive maintenance". Microelectronic Reliable, Vol .24, PP. 663-666, 1984

[5] El-Damcese, M.A., Shama,M.S. " reliability and availability analysis of a 2-state repairable system with two type of failure" Eng. Math. Lett, Vol .2015, 2015

[6] M.Yas. Haggag. "Profit Analysis and Availability of a repairable redundant3-out-of-4system involving Preventive Maintenance"International Journal of Scientific \& Engineering Research, Vol 6, 2015

[7] Ram, M. and Singh, S. B., "Analysis of a Complex System with common caus failure and two types of repair facilities with different distributions in failure"International Journal of Reliability and Safety, Vol. 4, pp. 381-392. (2010)

[8] Sachin kumar and Anand Tyagi, "Evaluation of some reliability parameters of three state repairable system with environmental failure"International Journal of Research and Reviews, Vol 1, 2009

[9] Singh, V. V, Rawal, D. K, "Availability, MTTF and Cost analysis of complex system under Preemptive resume repair policy using copula distribution"Pak.j.stat.oper.res ,Vol .X , pp299-312, 2014

[10] S.C. Agarwal, Mamta Sahani \& Shikha Bansal. "Reliability characteristic of cold standby redundant system" IJRRAS 3 (2), 2010. 